\title{
Dislocation Nucleation and Interaction under Nanoindentation in Single Crystalline Al and Cu: Molecular Dynamics Simulations
}

\author{
Tomohito TSURU $^{* *}$ and Yoji SHIBUTANI ${ }^{* * *}$ \\ ${ }^{* * N}$ Nuclear Science and Engineering Directorate, Japan Atomic Energy Agency, \\ 2-4 Shirakata-Shirane, Tokai-mura, Ibaraki 319-1195, Japan \\ E-mail: tsuru.tomohito@jaea.go.jp \\ ${ }^{* * *}$ Department of Mechanical Engineering, Graduate School of Engineering, Osaka University, \\ 2-1 Yamada-oka, Suita, Osaka, 565-0871, Japan \\ E-mail: sibutani@mech.eng.osaka-u.ac.jp
}

\begin{abstract}
Recent advances in miniaturization and highly-accurate measurement techniques have allowed mechanical properties to be measured at the nanometer scale. Nanoindentation has been widely used because of its applicability in ambient conditions. Unstable displacement burst or the abrupt growth of indent displacement after homogeneous elastic deformation observed in crystalline materials is a unique plastic deformation characteristic (nanoplasticity). In the present paper, a series of atomistic simulations of nanoindentation in single crystalline aluminum and copper are performed in analyzing the critical state for dislocation nucleation and interaction between dislocations beneath the indenter. With reference to the Hertzian solution based on isotropic linear elastic theory, both the anisotropic effect and nonlinear behavior of nanoindentation are discussed in detail. The discovery was made that the incipient yield process is strongly related to the triaxial stress state created beneath the indenter, and that energetically unfavorable interactions accompanied with cross slip induce the formation of prismatic dislocations.
\end{abstract}

Key words: Nanoindentation, Displacement Burst, Critical Resolved Shear Stress, Collective Dislocation Emission, Molecular Dynamics

\section{Introduction}

The nanoindentation technique was first proposed by Oliver and Pharr, and applied in evaluating the mechanical properties of nanometer-scale materials and thin films ${ }^{(1)}$. A specimen is indented using highly-accurate positioning with a very small load, providing the continuous relationship between indent load and depth. The mechanical properties of thin films can be derived using measured load-depth curves. Unlike uniaxial tensile testing, the indentation test produces a non-uniform stress distribution, whereby the local properties of the sample can be obtained with the expected resolution. An abrupt increase in the indent depth that occurs subsequent to homogeneous elastic deformation, or "displacement burst", can be observed in metallic materials below the $100-\mathrm{nm}$ scale of the indent depth ${ }^{(2)-(4)}$. And thus the fundamental deformation mechanism in nanoscale, which includes dislocation and other individual defect structures, affects the measuring response. Experimental observation via transmission electron microscopy (TEM) revealed high-density dislocation beneath the 
indenter ${ }^{(5)}$, thus enabling the prediction that collective dislocation nucleation and motion trigger the unexplained unstable behavior in nanoindentation.

Atomistic simulations have been developed with help of computation power, and enable direct simulations of the critical shear stress, the dislocation nucleation, and the dislocation motion around the spherical precipitation ${ }^{(6)}$. In the present study a sequence of atomistic simulations of nanoindentation in single-crystalline aluminum $(\mathrm{Al})$ and copper $(\mathrm{Cu})$ were performed in order to investigate the nucleation mechanism of dislocations in the defect-free region beneath the indentation. Referring to the Hertzian solution based on the isotropic linear elastic theory, the anisotropic effect, nonlinear behavior and dislocation nucleation with nanoindentation are discussed.

\section{Analysis model and method}

The atomic models used in the simulation are three-dimensional rectangular solids of different sizes containing up to 500,000 atoms (Model 1) and 2,048,000 atoms (Model 2) and used to investigate size dependence. The Cartesian coordinates used are $x[100], y[010]$, and $z[001]$, with indentation being implemented on the (001) plane. The indentation plane is traction-free with the atoms located at the bottom surface being fixed. Periodic boundary conditions are applied in the directions perpendicular to the indentation axis. An atomic model and boundary conditions are shown in Fig 1. The Embedded Atom Method (EAM)

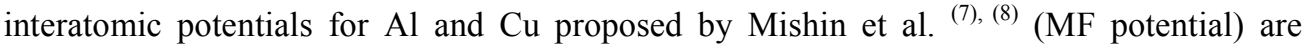
employed, and the repulsive potential by Kelchner et al. ${ }^{(9)}$ is used to simulate the frictionless contact between the atomic model and the spherical rigid indenter that provides the correspondence relationship with the Hertzian solution for the contact problem (10). Indentations are simulated using four different indenter radii $r$, and include $r=5,15,20$, and $30 \mathrm{~nm}$. In all the simulations quasi-static analysis at the temperature of absolute zero is performed using the Conjugate Gradient (CG) method. The indent load is applied iteratively in small increments $d z=0.025 \mathrm{~nm}$ of the indent depth, with the maximum depth being set at 1.5 to $2.0 \mathrm{~nm}$. A fully relaxed configuration for a given depth is obtained using the appropriate relaxation steps in the $\mathrm{CG}$.

\section{Mechanical properties under nanoindentation}

\subsection{Indent load-depth curves}

The relationships between indent load, mean pressure, and indent depth for indenters of varying radii are shown in Fig 2 . The mean pressure is defined as $p_{\mathrm{m}}=P / A_{\mathrm{c}}$, where $A_{\mathrm{c}}$ is the

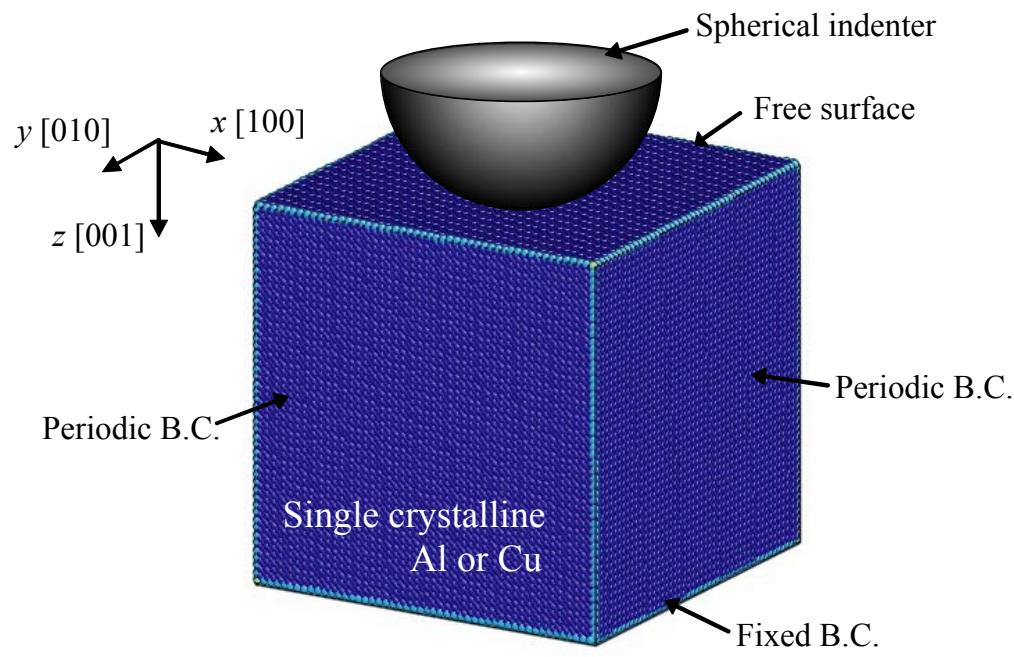

Fig. 1 Atomic model indented by spherical indenter. 


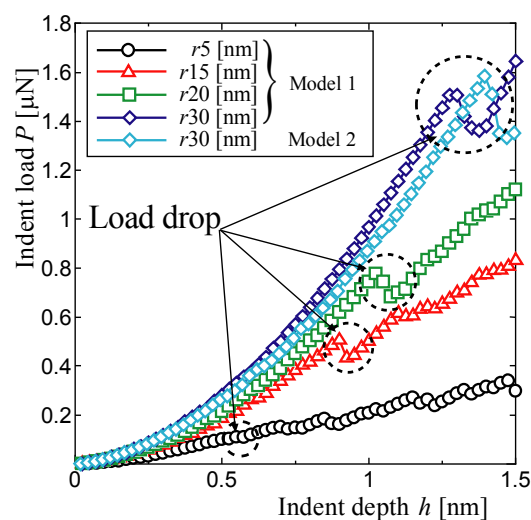

(a) Indent load - depth in $\mathrm{Al}$

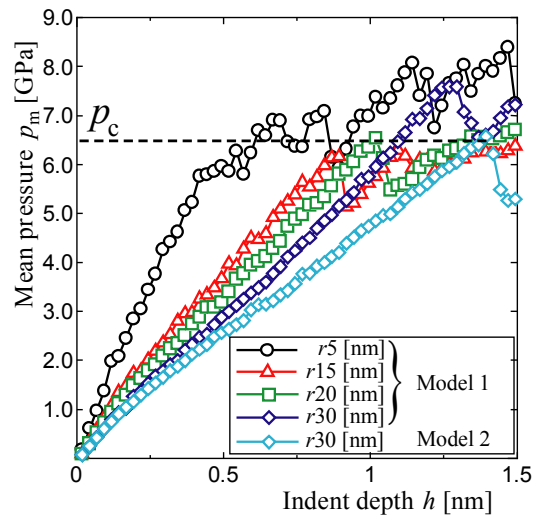

(c) Mean pressure - depth in $\mathrm{Al}$

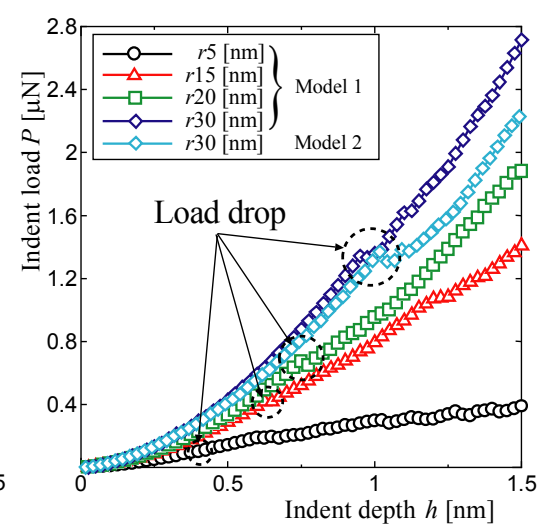

(b) Indent load - depth in $\mathrm{Cu}$

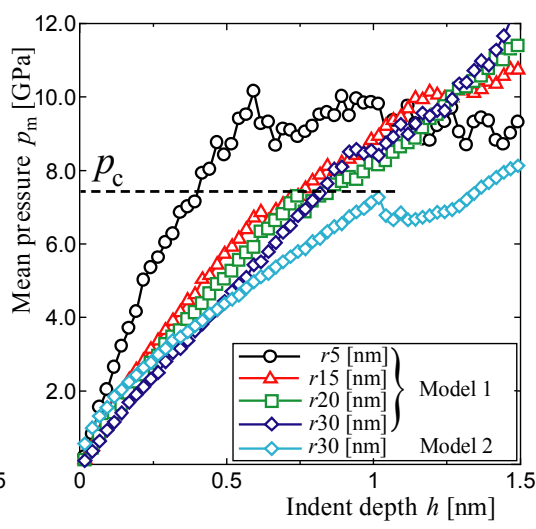

(d) Mean pressure - depth in $\mathrm{Cu}$

Fig. 2 Indent load and mean pressure with respect to indent depth.

contact area calculated directly from the atomic configurations when fully relaxed. Abrupt drops in both the indent load and mean pressure, indicative of dislocation nucleation, are observed after reversible elastic deformation (see the dashed circles in Fig 1 (a) and (b)), where the elastic behavior is in good agreement with the Hertzian solution of $P(h)=(4 / 3) E^{*} R^{1 / 2} h^{3 / 2}$. While critical drop load $P_{\mathrm{c}}$ increases with the radius of the indenter, critical mean pressure $p_{\mathrm{c}}$ remains at a specific value, apart from the smallest radius of indenter ( $r 5)$, which is independent of the radius. In Fig 2 (c) and (d), the critical pressures $p_{\mathrm{c}}$ are found to be 6.5 and $7.4 \mathrm{GPa}$ for $\mathrm{Al}$ and $\mathrm{Cu}$, respectively. It is known from the linear elastic solution for spherical Hertzian contact of isotropic bodies ${ }^{(10)}$ that a linear relationship exists between mean pressure and maximum shear stress, and can be expressed using the following equation:

$$
\tau_{\max }=0.31\left(\frac{6 P E^{* 2}}{\pi^{3} R^{3}}\right)^{\frac{1}{3}}=0.465 p_{\mathrm{m}} .
$$

According to the Hertzian solution critical shear stress $\tau_{c}^{\prime}$ below the indentation can be predicted using critical mean pressure. Substitution of the critical pressure obtained in atomistic simulations results in the critical shear stresses of 3.02 and 3.44 for $\mathrm{Al}$ and $\mathrm{Cu}$, respectively. This assessment technique has proved effective in predicting critical shear stress in experimental indentation tests ${ }^{(3),(11)}$. On the contrary, critical mean pressure is constant and can be solely determined with the material and indentation plane, provided the uniqueness of the critical shear stress under indentation can first be proved.

\subsection{Local stress condition under indentation}

Local stress based on atomic stress can be directly calculated using the present simulation. The coordinate system is transformed from the initial coordinate system of [001], [010], [001] into the $x^{\prime}[\overline{1} 10], y^{\prime}\left[1 \overline{2}^{2}\right], z^{\prime}[111]$ coordinate system through orthogonal 
transformation, with the atomic image of the $\{111\}\langle 11 \overline{2}\rangle$ shear stress distribution $\tau_{z^{\prime} y^{\prime}}$ just before dislocation emissions using Model 2 with an r30 indenter being given in Fig 3. We were able to confirm that maximum shear stress is generated in the inner region beneath the indenter, as predicted by the Hertzian elastic solution. However, the location is slightly removed from the indentation axis due to the inherent anisotropy of cubical crystal. The degree of eccentricity and distance from the surface are shown in Fig 4, where the position of maximum shear stress within the nearly perfect fcc structure is sought via the centrosymmetry parameter ${ }^{(9)}$. It was discovered that a one-to-one correspondence exists for all the cases except when the position is near the surface. It should be noted that while the distance from the surface given in Fig 4 (a) and (c) principally depend on the radius of the indenter, the degrees of eccentricity given in Fig 4 (b) and (d) differ with material. Anisotropic factors, evaluated using the following relationship of $\alpha=2 C_{44} /\left(C_{11}-C_{12}\right)$, were found to be 1.20 and 3.22 for $\mathrm{Al}$ and $\mathrm{Cu}$, with the difference deriving from the anisotropic

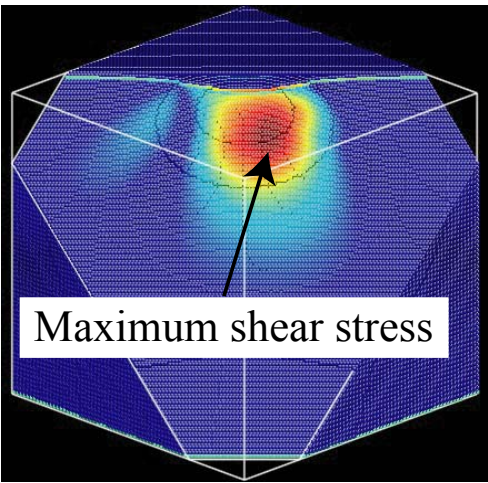

(a) $\mathrm{Al}$

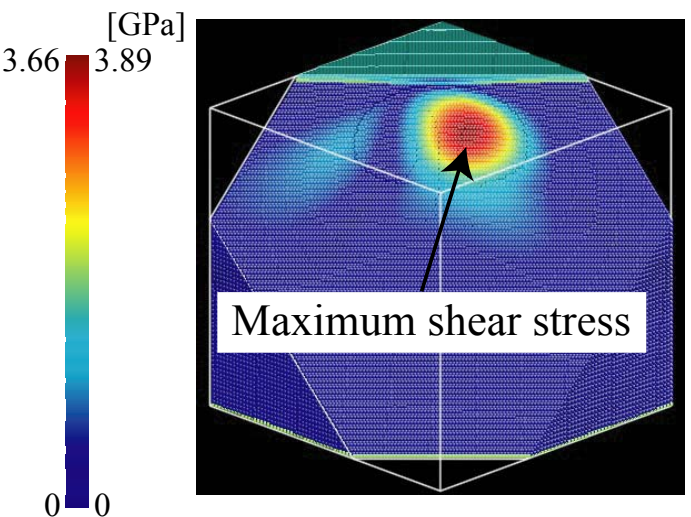

(b) $\mathrm{Cu}$

Fig. 3 Distribution of $\{111\}<112>$ shear stress.

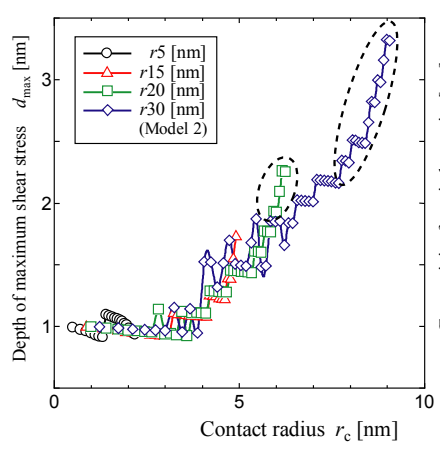

(a) Depth of $\tau_{\max }$ in $\mathrm{Al}$

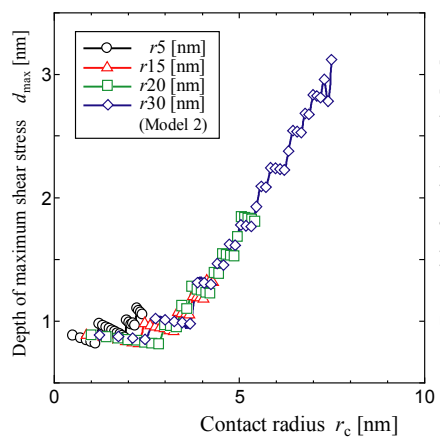

(c) Depth of $\tau_{\max }$ in $\mathrm{Cu}$

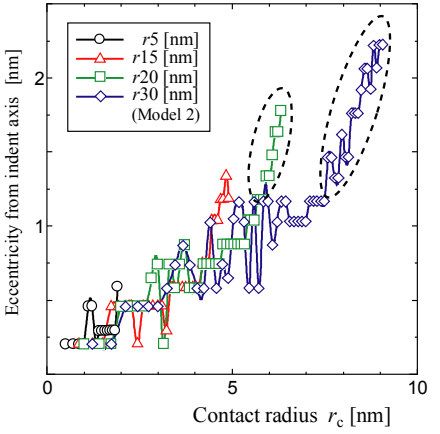

(b) Eccentricity from indent axis in $\mathrm{Al}$

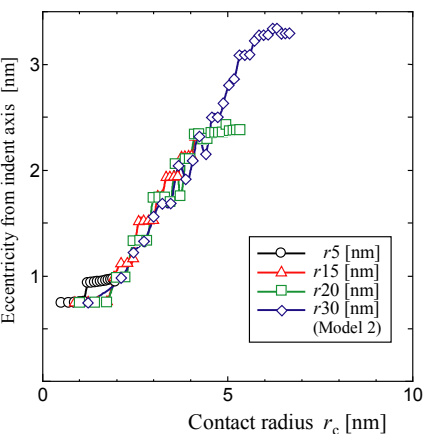

(d) Eccentricity from indent axis in $\mathrm{Cu}$

Fig. 4 Relationship between depth of maximum shear stress and contact radius. 
effect of the cubic crystals.

The Hertzian solution and the relationship between maximum shear stress and mean pressure calculated in the atomistic simulations are given in Fig 5. It was discovered that the critical value of maximum shear stresses provides the feature of being radius-independent for the relationship between mean pressure and depth, and a nearly-linear correlation for both $\mathrm{Al}$ and $\mathrm{Cu}$. Conversely, the gradient obtained using least squares approximation differs for $\mathrm{Al}$ and $\mathrm{Cu}(0.750$ for $\mathrm{Al}$ and 0.617 for $\mathrm{Cu})$. Both are much larger than the Hertzian solution (0.465), with $\mathrm{Al}$ in particular being more apparent than $\mathrm{Cu}$. It is known that actual materials deform nonlinearly depending on the triaxial stress conditions ${ }^{(12)}$. Hence the internal stress conditions beneath the indenter were then considered. The evolution of the maximum $\{111\}\langle 11 \overline{2}\rangle$ shear stress, as well as the normal stress components of $\sigma_{x^{\prime} x^{\prime}}, \sigma_{y^{\prime} y^{\prime}}$, $\sigma_{z^{\prime} z^{\prime}}$, and hydrostatic pressure $p_{\mathrm{h}}$ at the point of maximum shear stress for Model 1 indented with $r 15$ and Model 2 with $r 30$, are depicted in Fig 6 . The magnitude of all the normal stresses and hydrostatic pressure gradually increase on the compressed side with increased

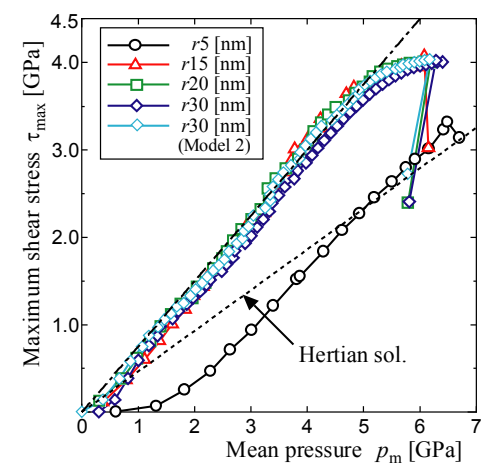

(a) Al

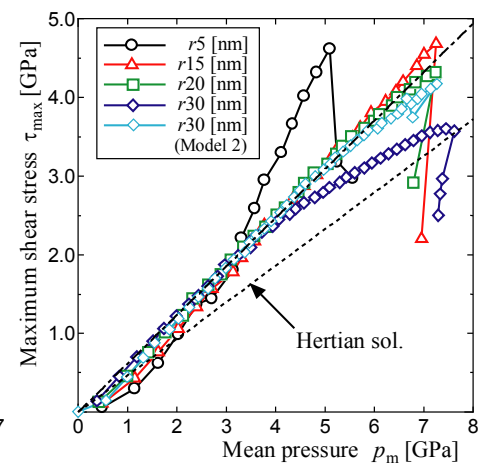

(b) $\mathrm{Cu}$

Fig. 5 Relationship between maximum shear stress and mean pressure.

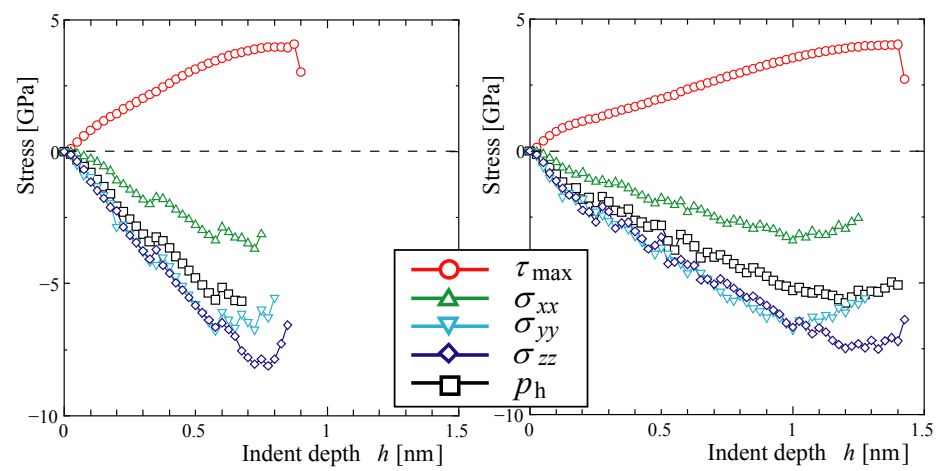

(a) $\mathrm{Al}: r 15 \mathrm{~nm}$

(b) $\mathrm{Al}: r 30 \mathrm{~nm}$

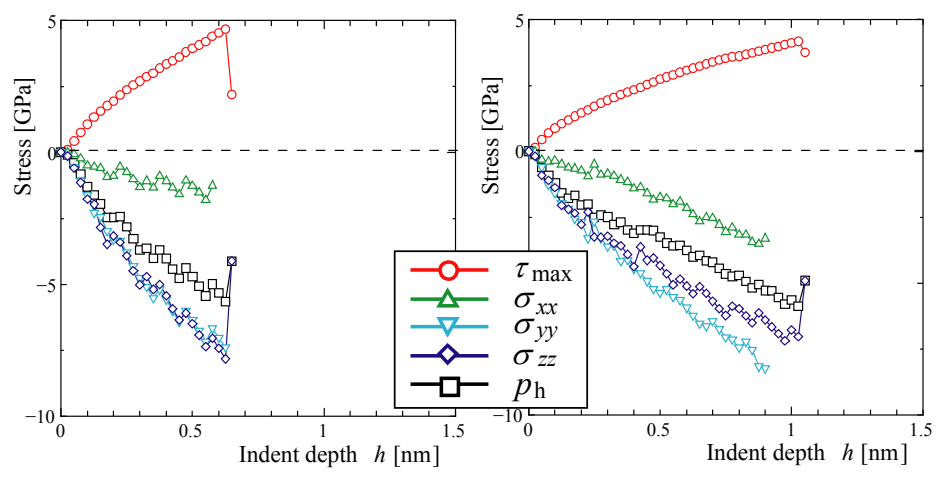

(c) $\mathrm{Cu}: r 15 \mathrm{~nm}$

(d) $\mathrm{Cu}: \mathrm{r} 30 \mathrm{~nm}$

Fig. 6 Maximum $\{111\}\langle 11 \overline{2}\rangle$ shear stress as well as normal stress with respect to indent depth. 
indent depth. It was discovered in all cases that the hydrostatic pressure reaches $-5 \mathrm{GPa}$ just prior to the dislocation emissions. In addition it is particularly worth noting that the critical shear stress and normal stresses provide the radius-independent values. In order to implement more rigorous calculations, atomic stress was evaluated using the Voronoi volume at the atomic position of the maximum shear stress. Voronoi volumes for $\mathrm{Al}$ and $\mathrm{Cu}$ were found to be 94.8 and 97.6 percent of the perfect crystal volume under zero stress, thus providing definite evidence that local-compressive stress states are present in indentation. Accurate critical stress and maximum normal stresses are given in Table 1. Here the ideal shear strengths for $\mathrm{Al}$ and $\mathrm{Cu}$ using the MF potential were preliminarily evaluated: When normal stresses are unrelaxed, the shear strength $\tau_{\mathrm{c}}^{u}=3.60$ at $3.91 \mathrm{GPa}$, and when relaxed $\tau_{\mathrm{c}}^{r}=3.19$ at $2.86 \mathrm{GPa}$, for $\mathrm{Al}$ and $\mathrm{Cu}$, respectively ${ }^{(13)}$. The critical shear stresses under indentation are of much higher values than these shear strengths, being 15.2 and 10.8 percent of the shear moduli for $\mathrm{Al}$ and $\mathrm{Cu}$, respectively. This remarkable discrepancy is caused by the normal stress component. Recent $a b$ initio calculations indicated pressure dependence of the critical shear strength, and elucidated that triaxial compressive stress causes the critical shear stress to increase ${ }^{(12)}$. It was confirmed that high compressive pressure is generated with indentation.

\section{Dislocation emission and interaction}

An overview of the dislocation emissions under nanoindentation in $\mathrm{Al}$ and $\mathrm{Cu}$ achieved by atomistic simulations using Model 2 and r30 indenter are given in Fig 7, with the images

Table 1 Maximum shear stress and normal stress at the moment of dislocation nucleation.

\begin{tabular}{ccccc}
\hline & Maximum shear stress [GPa] & \multicolumn{3}{c}{ Normal stress [GPa] } \\
\hline \hline & $\tau_{\mathrm{c}}$ & $\sigma_{x^{\prime} x^{\prime}}$ & $\sigma_{y^{\prime} y^{\prime}}$ & $\sigma_{z^{\prime} z^{\prime}}$ \\
$\mathrm{Al}$ & 4.24 & -2.13 & -5.23 & -7.55 \\
$\mathrm{Cu}$ & 4.42 & -2.89 & -8.04 & -7.93 \\
\hline
\end{tabular}

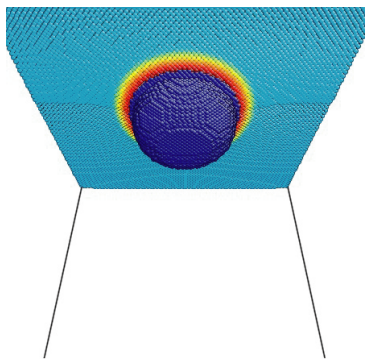

(i) $d=1.375 \mathrm{~nm}$

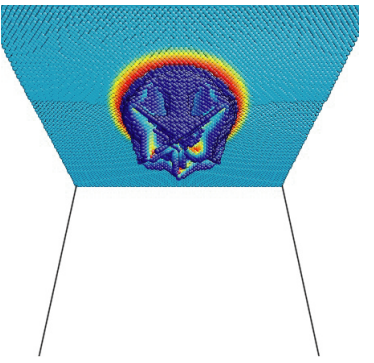

(ii) $d=1.425 \mathrm{~nm}$

(a) $\mathrm{Al}$

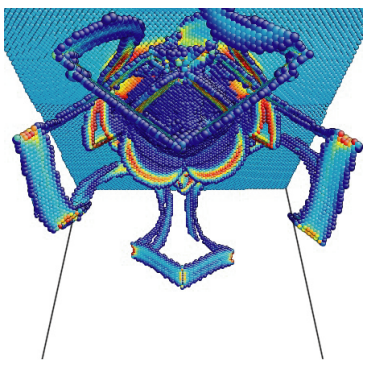

(iii) $d=1.750 \mathrm{~nm}$

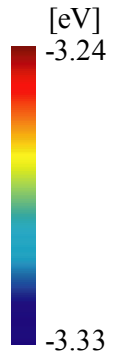

$-3.33$

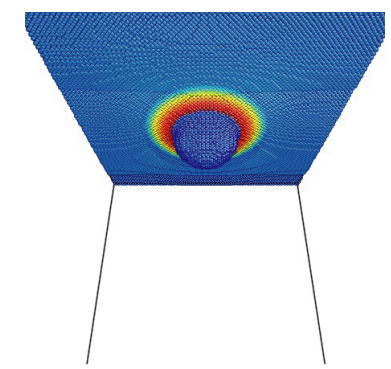

(i) $d=1.000 \mathrm{~nm}$

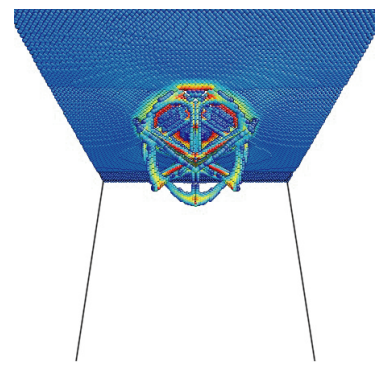

(ii) $d=1.050 \mathrm{~nm}$

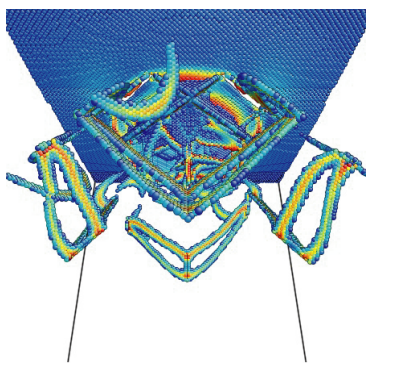

(iii) $d=1.500 \mathrm{~nm}$

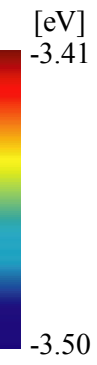

(b) $\mathrm{Cu}$

Fig. 7 Atomic image of dislocation emission in $\mathrm{Al}$ and $\mathrm{Cu}$ indented by $\mathrm{r} 30 \mathrm{~nm}$ spherical indenters. 
having been created with the visualization software AtomEye ${ }^{(14)}$. We extracted the specific high energy structures on the basis of potential energy criteria and specifying only the atoms with energy above $-3.33 \mathrm{eV}$ for $\mathrm{Al}$ and $-3.50 \mathrm{eV}$ for $\mathrm{Cu}$. The first image for each material shows the elastic deformation, wherein the atoms of high strain energy can be observed. The dislocations are then emitted within the material. The indication is that high elastic strain energy is converted into the total dislocation energy. And once the dislocations have nucleated they then interact with the dislocations on the other slip plane. This interaction process has been investigated in a large number of atomistic simulations, and it is presumed that Lomer-Cottrell type sessile dislocations are generated (15), (16). However, when investigating the interaction between dislocations the reaction energy both before and after the interaction needs to be examined. Based on the classical dislocation theory, the square of Burgers vector for two dislocations is considered. Stable reactions for two partial dislocations using Thompson notation ${ }^{(17)}$ are listed in Table 2. For fcc metals there are 12 slip systems, and eight of them are activated in nanoindentation because the slip system parallel to the indentation surface is excluded. For example, dislocations with $\boldsymbol{b}_{\mathrm{A}}$ on $(11 \overline{1})$ and $\boldsymbol{b}_{\mathrm{B}}$ on $(1 \overline{1} 1)$ are particularly worthy of note. The selected dislocations dissolved into the following partial dislocations:

$$
\begin{aligned}
& \boldsymbol{b}_{A} \rightarrow \boldsymbol{b}_{A}^{\mathrm{LP}}+\boldsymbol{b}_{A}^{\mathrm{TP}}: \frac{a}{2}[011] \rightarrow \frac{a}{6}[112]+\frac{a}{6}[\overline{1} 21] \\
& \boldsymbol{b}_{B} \rightarrow \boldsymbol{b}_{B}^{\mathrm{LP}}+\boldsymbol{b}_{B}^{\mathrm{TP}}: \frac{a}{2}[011] \rightarrow \frac{a}{6}[121]+\frac{a}{6}[\overline{1} 12]
\end{aligned},
$$

where the suffixes LP and TP denote the Leading and Trailing Partial dislocations, respectively. The square of the dissociated partials after the interaction, $\left(\boldsymbol{b}_{A}^{\mathrm{LP}}+\boldsymbol{b}_{B}^{\mathrm{LP}}\right)^{2}$ and $\left(\boldsymbol{b}_{A}^{\mathrm{TP}}+\boldsymbol{b}_{B}^{\mathrm{TP}}\right)^{2}$, are found to be $22 \times\left(a^{2} / 36\right)$, which is larger than before the interaction. And hence revealing that the interaction of dislocations in indentation is unstable and the sessile dislocation never formed. Afterwards the formation of a Prismatic Dislocation Loop (PDL) was observed, and is given in Fig 8. PDLs form because of the unstable interaction between dislocations and cross slips, as previously reported by the authors ${ }^{(13)}$. We confirmed that PDLs form within the radial stress distribution generally produced by indentation or inclusion causing multiple slips.

\section{Conclusions}

Dislocation emissions and their interaction in nanoindentation were investigated via atomistic simulations. In the present study different radii spherical indenters were used for the purpose of ensuring an in depth investigation was made. It was discovered that the calculated critical mean pressure and critical shear stress for the dislocation emission provide radius-independent but equivalent values. The maximum shear stress is generated in the inner region beneath the indenter, as predicted by the Hertzian elastic solution, but slightly removed from the indentation axis due to inherent anisotropy. In addition, a considerably high compressive pressure is generated and causes an elevation in the critical shear stress for dislocation emissions. Finally, it was confirmed that the emitted dislocation in nanoindentation causes the unstable interactions with dislocation on the other slip system,

Table 2 Stable reactions between two partial dislocations expressed in Thompson notation.

\begin{tabular}{cccc}
\hline $\begin{array}{c}\text { Reaction } \\
\text { number }\end{array}$ & $\begin{array}{c}\text { Stair-rod } \\
\boldsymbol{b}_{3}\end{array}$ & $\begin{array}{c}\text { Thompson notation } \\
\boldsymbol{b}_{1}+\boldsymbol{b}_{2}=\boldsymbol{b}_{3}\end{array}$ & $\begin{array}{c}\boldsymbol{b}_{3}{ }^{2} \\
\left(\times a^{2} / 36\right)\end{array}$ \\
\hline \hline 1 & $(a / 6)<110>$ & $D \alpha+B \gamma=D B / \alpha \gamma$ & 2 \\
2 & $(a / 6)<200>$ & $\alpha B+B \gamma=\alpha \gamma$ & 4 \\
3 & $(a / 6)<220>$ & $C \alpha+A \gamma=C A / \alpha \gamma$ & 8 \\
4 & $(a / 6)<310>$ & $C \alpha+\gamma D=C \gamma / \alpha D$ & 10 \\
\hline
\end{tabular}



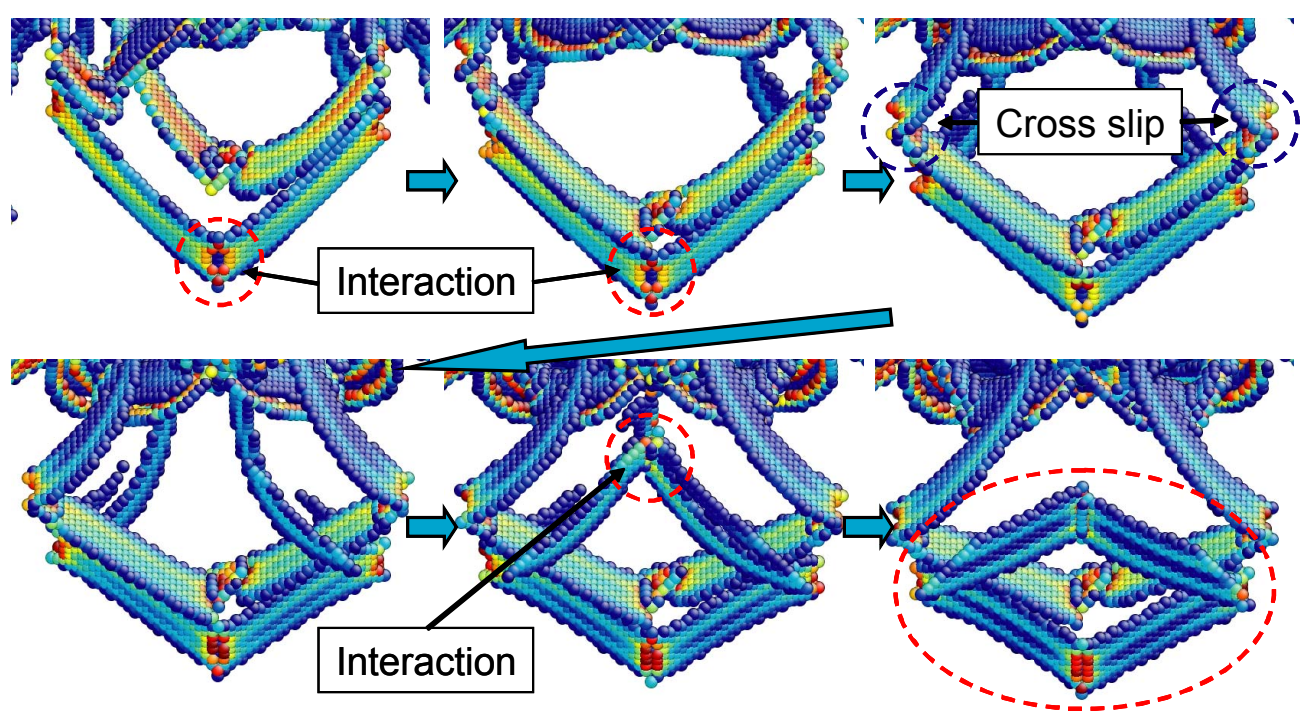

Fig. 8 Formation of a prismatic dislocation loop under nanoindentation.

which never form sessile dislocations. And as a result rhombic prismatic dislocation loops form because of the unstable interactions and cross slips.

\section{Acknowledgement}

The authors would like to acknowledge the financial support of the Japan Society for the Promotion of Science (JSPS), Grant-in-Aid for JSPS Fellows (179504)(TT), Young Scientists (Start-up, 19860088)(TT), and Scientific Research (S, 20226004)(YS).

\section{References}

(1) Oliver, W. C. and Pharr, G. M., An improved technique for determination hardness and elastic modulus using load and displacement sensing indentation experiments, Journal of Materials Research, Vol. 7, No. 6 (1992), pp. 1564-1583.

(2) Gerberich, W. W., Nelson, J. C., Lilleodden, E. T., Anderson, P. and Wyrobek, J. T., Indentation induced dislocation nucleation: The initial yield point, Acta Materialia, Vol. 44, No. 9 (1996), pp. 3585-3598.

(3) Suresh, S., Nieh, T.-G. and Choi, B. W., Nano-indentation of copper thin films on silicon substrates, Scripta Materialia, Vol. 41, No. 9 (1999), pp. 951-957.

(4) Shibutani, Y. and Koyama, A., Surface roughness effects on the displacement bursts observed in nanoindentation, Journal of Materials Research, Vol. 19, No. 1 (2004), pp. 183-188.

(5) Robertson, C. F. and Fivel, M. C., A study of the submicron indent-induced plastic deformation, Journal of Materials Research, Vol. 14, No. 6 (1999), pp. 2251-2258.

(6) Tsuru, T. and Shibutani, Y., Formation and critical shear stresses of prismatic dislocation loops observed around spherical precipitate in single crystalline aluminum and copper matrices (in Japanese), Transactions of the Japan Society of Mechanical Engineers, Series $A$, Vol. 71, No. 711 (2005), pp. 1445-1450.

(7) Mishin, Y., Farkas, D., Mehl, M. J. and Papaconstantopoulos, D. A., Interatomic potentials for monoatomic metals from experimental data and ab initio calculations, Physical Review B, Vol. 59, No. 5 (1999), pp. 3393-3407.

(8) Mishin, Y., Mehl, M. J., Papaconstantopoulos, D. A., Voter, A. F. and Kress, J. D., Structural stability and lattice defects in copper: $\mathrm{Ab}$ initio, tight-binding, and embedded-atom calculations, Physical Review B, Vol. 63, No. 22 (2001), pp. 224106.

(9) Kelchner, C. L., Plimpton, S. J. and Hamilton, J. C., Dislocation nucleation and defect structure during surface indentation, Physical Review B, Vol. 58, No. 17 (1998), pp. 
11085-11088.

(10) Johnson, K. L., Contacts Mechanics, Cambridge University Press, Cambridge, 2003 (First published 1985).

(11) Gouldstone, A., Koh, H.-J., Zeng, K.-Y., Giannakopoulos, A. E. and Suresh, S., Discrete and continuous deformation during nanoindentation of thin films, Acta Materialia, Vol. 48, No. 9 (2000), pp. 2277-2295.

(12) Ogata, S., Li, J. and Yip, S., Ideal pure shear strength of aluminum and copper, Science, Vol. 298, No. 25 (2002), pp. 807-811.

(13) Tsuru, T. and Shibutani, Y., Atomistic simulations of elastic deformation and dislocation nucleation in $\mathrm{Al}$ under indentation-induced stress distribution, Modelling and Simulation in Materials Science and Engineering, Vol. 14, No. 5 (2006), pp. S55-S62.

(14) Li, J., AtomEye: An efficient atomistic configuration viewer, Modelling and Simulation in Materials Science and Engineering, Vol. 11, No. 2 (2003), pp. 173-177.

(15) Gannepalli, A. and Mallapragada, S. K., Atomistic studies of defect nucleation during nanoindentation of $\mathrm{Au}(001)$, Physical Review B, Vol. 66, No. 10 (2002), pp. 104103.

(16) Liang, H. Y., Woo, C. H., Huang, H., Ngan, A. H. W. and Yu, T. X., Dislocation nucleation in the initial stage during nanoindentation, Philosophical Magazine, Vol. 83, No. 31 (2003), pp. 3609-3622.

(17) Hirth, J. P. and Lothe, J., Theory of Dislocations, 2nd edition, Wiley, New York, 1982. 